\title{
Entrainment of the Fetal Hamster Circadian Pacemaker by Prenatal Injections of the Dopamine Agonist SKF 38393
}

\author{
N. Viswanathan, ${ }^{1}$ D. R. Weaver, ${ }^{2}$ S. M. Reppert, ${ }^{2}$ and F. C. Davis ${ }^{1}$ \\ 'Department of Biology, Northeastern University, Boston, Massachusetts 02115, and 'Laboratory of Developmental \\ Chronobiology, Massachusetts General Hospital and Harvard Medical School, Boston, Massachusetts 02114
}

Prenatal treatment with the $D_{1}$-dopamine receptor agonist SKF 38393 or cocaine induces expression of the immediateearly gene $c$-fos in the fetal rat suprachiasmatic nucleus (SCN) (Weaver et al., 1992). Because the induction of $c$-fos gene expression in the SCN has been implicated in the entrainment of circadian rhythms by light in mature animals, the present study investigated whether prenatal dopaminergic activation entrains the fetal circadian pacemaker. Injections of SKF $38393(8 \mathrm{mg} / \mathrm{kg}$ ) were given to pregnant, SCN-lesioned hamsters during the last $5 \mathrm{~d}$ of gestation and the phases of the offspring's wheel-running activity rhythms were measured on postnatal day 20. Pregnant hamsters were each given two injections/day $12 \mathrm{hr}$ apart, but only one of the injections each day contained SKF 38393. One group of hamsters received the drug at $0800 \mathrm{hr}$ while another group received the drug at $2000 \mathrm{hr}$. The offspring from these treatment groups showed average phases that differed by 11.3 hr, demonstrating that prenatal SKF 38393 set the phase of the offspring's circadian rhythms. These results suggest that the fetal circadian pacemaker can be entrained by dopaminergic activation. In situ hybridization using cRNA probes demonstrated that a single injection of SKF 38393 on the last day of gestation induced $c$-fos gene expression in the fetal hamster SCN and that mRNA for the $D_{1}$-dopamine receptor was present in the SCN at that time. It is possible that maternal entrainment of the fetal circadian pacemaker, which normally occurs during development, is mediated by dopaminergic activation within the fetal hypothalamus.

IKey words: suprachiasmatic nucleus, circadian, dopamine, SKF 38393, D1 receptor, hamster, development, hypothalamus]

The physiology and behavior of animals are regulated by endogenously generated rhythms with periods of approximately $24 \mathrm{hr}$ (circadian). In mammals, these rhythms are regulated by a circadian pacemaker within the suprachiasmatic nucleus (SCN) of the hypothalamus (Klein et al., 1991). Evidence that a pace-

\footnotetext{
Received Sept. 27, 1993; revised Jan. 25, 1994; accepted Mar. 9, 1994.

This work was supported by National Institutes of Health Grant HD18686 to F.C.D. and HD14427 to S.M.R.

Correspondence should be addressed to Fred C. Davis, Department of Biology, 414 Mugar Building, Northeastern University, Boston, MA 02115.

Copyright (C) 1994 Society for Neuroscience $0270-6474 / 94 / 145393-06 \$ 05.00 / 0$
}

maker is located within the SCN includes results showing that ablation of the SCN disrupts circadian rhythms (Moore and Eichler, 1972; Stephan and Zucker, 1972) and that transplantation of fetal hypothalamus containing the SCN to the brain of an SCN-ablated adult restores circadian rhythms with timing properties characteristic of the donor (Ralph et al., 1990). Although circadian rhythms are endogenously generated, they are normally entrained to daily rhythmicity in the environment. Circadian rhythms thereby assume characteristic and adaptive phase relationships to predictable events in the environment (Pittendrigh, 1981). For mature organisms, the most important entraining signal is the environmental light/dark cycle. Photic information is detected by retinal photoreceptors and reaches the SCN via direct and indirect retinal projections (Moore and Card, 1985).

During development, the pacemaker begins to function before photic information is able to cause entrainment (Reppert and Weaver, 1991). In rats and hamsters, the pacemaker begins to generate oscillations before birth and these oscillations are entrained by circadian rhythms of the dam. Rhythms such as the activity/rest rhythm and a rhythm in activity of the pineal gland enzyme $N$-acetyltransferase are not expressed until after birth, but the phase of these rhythms can be set by maternal rhythmicity before birth (Deguchi, 1975; Reppert et al., 1984; Davis and Gorski, 1986). Ablation of the maternal SCN in the first half of gestation disrupts entrainment of the offspring, indicating that a maternal signal regulated by the SCN mediates entrainment (Reppert and Schwartz, 1986a; Davis and Gorski, 1988). Although the specific signal or signals normally responsible for prenatal entrainment have not been identified, two maternal rhythms have been implicated with studies employing SCNlesioned dams. In hamsters, daily injections of the pineal gland hormone melatonin sets the phase of the pups' activity/rest rhythms (Davis and Mannion, 1988; Viswanathan and Davis, 1993), and in rats a restricted feeding schedule imposed on the dam sets the phase of the pups' drinking rhythm (Weaver and Reppert, 1989).

Recent evidence suggests that activation of a dopaminergic system within the fetus may be involved in the mechanism of prenatal entrainment. Injections of the $D_{1}$-dopamine receptor agonist SKF 38393 or cocaine to pregnant rats induce expression of mRNA for the proto-oncogene $c$-fos in the fetal SCN (Weaver et al., 1992). Because the induction of $c$-fos has been implicated in entrainment of the circadian pacemaker by light in adults 
(Takahashi et al., 1993), it is possible that the induction of $c$-fos expression in the fetal SCN also causes entrainment. The present experiments were undertaken to test the hypothesis that prenatal activation of a dopaminergic system can entrain the fetal pacemaker. We report that $D_{1}$-dopamine receptor $m R N A$ is present within the fetal hamster $S C N$, that the $D_{1}$-dopamine receptor agonist SKF 38393 induces $c$ - fos gene expression in the fetal hamster SCN (as it does in rats), and, importantly, that prenatal injections of SKF 38393 entrain the hamster circadian pacemaker.

\section{Materials and Methods}

General methods. Eight-week-old Syrian hamsters, Mesocricetus auratus (LVG), from Charles River Laboratories (Kingston, NY) were kept individually under a 14:10 hr light/dark cycle (lights off from 2200 to $0800 \mathrm{hr}$ ). Estrous cycles of female hamsters were determined by daily monitoring of the vaginal discharge. The females were paired with males overnight on the night of ovulation. For the designation of embryonic ages, fertilization was assumed to occur midway through the dark period so that 0300 on the night of pairing was E0. On the day after pairing the females were transferred to dim constant light (LL, $<1$ lux) and housed in cages equipped with a running wheel. Food and water were available at all times.

On day 7 of gestation the hamsters received lesions directed at the suprachiasmatic nuclei (SCN). Hamsters were anesthetized with sodium pentobarbital $(100 \mathrm{mg} / \mathrm{kg}$ body weight $)$ and placed in a stereotaxic instrument. A midline incision was made on the head and a hole of $\sim 2$ $\mathrm{mm}$ diameter was drilled at the midline of the skull $0.6 \mathrm{~mm}$ anterior to bregma. A platinum-iridium (90:10) wire electrode (anode) in a 26 gauge stainless steel cannula coated with epoxy resin insulation except for $0.3 \mathrm{~mm}$ of the wire tip was lowered through the hole to a point 8.4 $\mathrm{mm}$ below the skull surface. A needle (cathode) was placed subcutaneously and a current of $4 \mathrm{~mA}$ was passed for $8 \mathrm{sec}$. The hole was filled with surgical foam and the incision was closed with surgical staples. The pregnant, SCN-lesioned hamsters subsequently received one or more intraperitoneal injections of the $D_{1}$-dopamine receptor agonist SKF 38393 (8 mg/kg; Research Biochemicals, Natick, MA), injected in a volume of $0.5 \mathrm{ml}$ of $0.9 \%$ saline.

Histological analysis was performed to confirm ablations of the SCN. Hamsters were killed either several weeks after weaning of their litters (experiment 1 ) or on day 15 of gestation (experiment 2 ). The latter were killed by decapitation and the brain (except a portion of forebrain frozen for in situ hybridization) was immersed in $10 \%$ formalin. The hamsters killed after weaning were anesthetized with sodium pentobarbital and intracardially perfused with phosphate $(0.01 \mathrm{M})$-buffered saline with heparin $(25,000 \mathrm{U} /$ liter $)$, followed by $10 \%$ formalin. The brains were removed and postfixed in $10 \%$ formalin for at least $24 \mathrm{hr}$. The brains from all of the lesioned hamsters were kept overnight in $20 \%$ ethanol for cryoprotection before sectioning. Frozen microtome sections $(90$ $\mu \mathrm{m})$ were cut through the SCN region and stained with thionin. Serial sections through the $\mathrm{SCN}$ region were used to estimate the extent and location of lesions.

Experiment 1: circadian rhythms. To test the hypothesis that prenatal injections of SKF 38393 entrain the fetal circadian pacemaker, the phases of hamster pup circadian rhythms were measured following prenatal injections of SKF 38393. Injections of SKF 38393 were given to pregnant, SCN-lesioned hamsters maintained in dim LL so that pups could not receive time-of-day information except from the timed injections. Wheel-running behavior was recorded from the $\mathrm{SCN}$-lesioned hamsters in order to evaluate the completeness of SCN ablations. Visual inspection of such records were used to select hamsters and their litters for inclusion in the study. The extent of SCN lesions was later determined by histological analysis. Ten pregnant, SCN-lesioned hamsters received two intraperitoneal injections each day $12 \mathrm{hr}$ apart on days 11-15 of gestation. One group received SKF 38393 in the morning $(0800, n=4)$ and another group received it in the evening $(2000, n=$ 6). In both groups, vehicle only was given at the time when SKF 38393 was not administered. Following the last injection, the hamsters and their litters (born the following day) were left undisturbed until the pups were weaned on postnatal day 20 . At weaning, pups from the A.M. ( $n$ $=27)$ and P.M. $(n=35)$ groups (at least five pups per litter regardless of sex) were separated from the dams and immediately introduced into individual running-wheel cages, still maintained in dim LL. Weaning was performed at two different times of day, $12 \mathrm{hr}$ apart $(0800,2000)$, to assess any possible effect of weaning on the phases of pups' activity rhythms. The free-running activity rhythms of hamster pups were continuously recorded for 3-4 weeks after weaning, and the phases of activity onset on the day of weaning were calculated from these rhythms. The phase of the wheel-running activity rhythm provides a reliable estimate of the phase of the underlying circadian pacemaker within the SCN (Davis, 1989).

The number of wheel revolutions within $10 \mathrm{~min}$ bins was continuously collected and stored using a Dataquest III system (Mini-mitter Co., Inc., Sunriver, OR). Activity records were subsequently analyzed and graphically displayed using Circadia software (Behavioral Cybernetics, Cambridge, MA). The circadian free-running period was estimated for each pup with the $\chi^{2}$ periodogram (Sokolov and Bushell, 1978). The activity record was then folded at the appropriate period to generate an average waveform. All pups showed an average waveform in which approximately half of the circadian cycle consisted of little or no activity and the other half showed continuous or episodic activity. The phase of activity onset was taken as the midpoint of the initial rise in activity at the beginning of the active portion of the cycle (Viswanathan and Davis, 1993). Phases were plotted on a circle representing the $24 \mathrm{hr}$ of postnatal day 20 . The average phase on day 20 was calculated by vector addition, and the scatter among the values was represented by the length $(r)$ of the average vector. Whether a given distribution was significantly different from uniform $(p<0.05)$ was determined by the Rayleigh test (Batschelet, 1981), and whether there was a significant difference between two groups of phases $(p<0.05)$ was determined by the Mardia, Watson, and Wheeler test (Batschelet, 1981).

Experiment 2: in situ hybridization. Single intraperitoneal injections of the $D_{1}$-dopamine receptor agonist SKF 38393 were given to pregnant, SCN-lesioned hamsters on day 15 of gestation in either the morning $(0800, \mathrm{E} 14.25, n=3)$ or evening $(2000, \mathrm{E} 14.75, n=2)$. The same number of hamsters at each time received saline vehicle only. Forty minutes following injection, hamsters were killed by decapitation in dim constant light. Fetal heads and adult forebrains were frozen in 2-methylbutane $\left(-20^{\circ} \mathrm{C}\right)$ and stored at $-80^{\circ} \mathrm{C}$ until sectioning. Serial, $15 \mu \mathrm{m}$ sections through the SCN were cut using a cryostat and were thaw-mounted on slides coated with Vectabond (Vector Laboratories). Sections were distributed to slides in a 1 -in-6 series so that sections on each slide were spaced at $90 \mu \mathrm{m}$ intervals. Slides were air dried and stored at $-80^{\circ} \mathrm{C}$. Three to five fetuses from each litter were examined.

In situ hybridization was performed as previously described (Reppert et al., 1991). The specificity of hybridization signals was estimated by comparing adjacent sections hybridized with the antisense and sense (control) cRNA probes produced from the same plasmids.

A plasmid containing the 2141 nucleotide mouse $c$-fos $\mathrm{CDNA}$ in the vector pGEM-4 (Promega, Madison, WI) was provided by M. E. Greenberg (Harvard Medical School, Boston, MA). Antisense and sense complementary RNA (cRNA) probes were produced by linearization with the appropriate restriction endonucleases, $\mathrm{Bgl} \mathrm{II}$ or Bam $\mathrm{HI}$, followed by in vitro transcription with T7 or SP6 RNA polymerase (Promega) in the presence of Uridine $5^{\prime}-\left[\alpha-\left({ }^{35} \mathrm{~S}\right)\right.$ thio $]$ triphosphate $(1100-1400 \mathrm{Ci} /$ mmol; New England Nuclear, Boston, MA). The human $D_{1}$-dopamine receptor cDNA in a pRC/CMV vector (Invitrogen, San Diego) was provided by J. S. Fink (Massachusetts General Hospital, Boston, MA). An $840 \mathrm{bp}$ Pst I-Hind III fragment corresponding to nucleotides -237 to +602 , where 1 is the A of the ATG encoding the initiator methionine, was subcloned to Bluescript II SK + (Stratagene). The antisense and sense probes were produced by linearization with Xba I or Hind III followed by transcription with T7 or T3 RNA polymerases. Preliminary studies indicated that this human $D_{1}$ receptor probe gave a more intense hybridization signal in the adult hamster caudate-putamen than did the rat probe used previously (Weaver et al., 1992).

Hybridization signals were detected using film autoradiography, and signals following hybridization with the $c$-fos $\mathrm{cRNA}$ probe were quantified by optical densitometry. From each fetal brain, one to three sections containing the SCN were used for densitometry. The optical densities (OD) of the fetal SCN and adjacent hypothalamus were determined using the Drexel University Image Processing Center "Brain" software package run on an IBM-AT computer. Optical density values were within the linear response range of the film. The relative OD of SCN (OD of SCN/OD of adjacent hypothalamus) was calculated for each section and the values from different sections from each fetus were averaged. In contrast to the SCN, treatment-related hybridization signal was not seen in adjacent hypothalamus. 

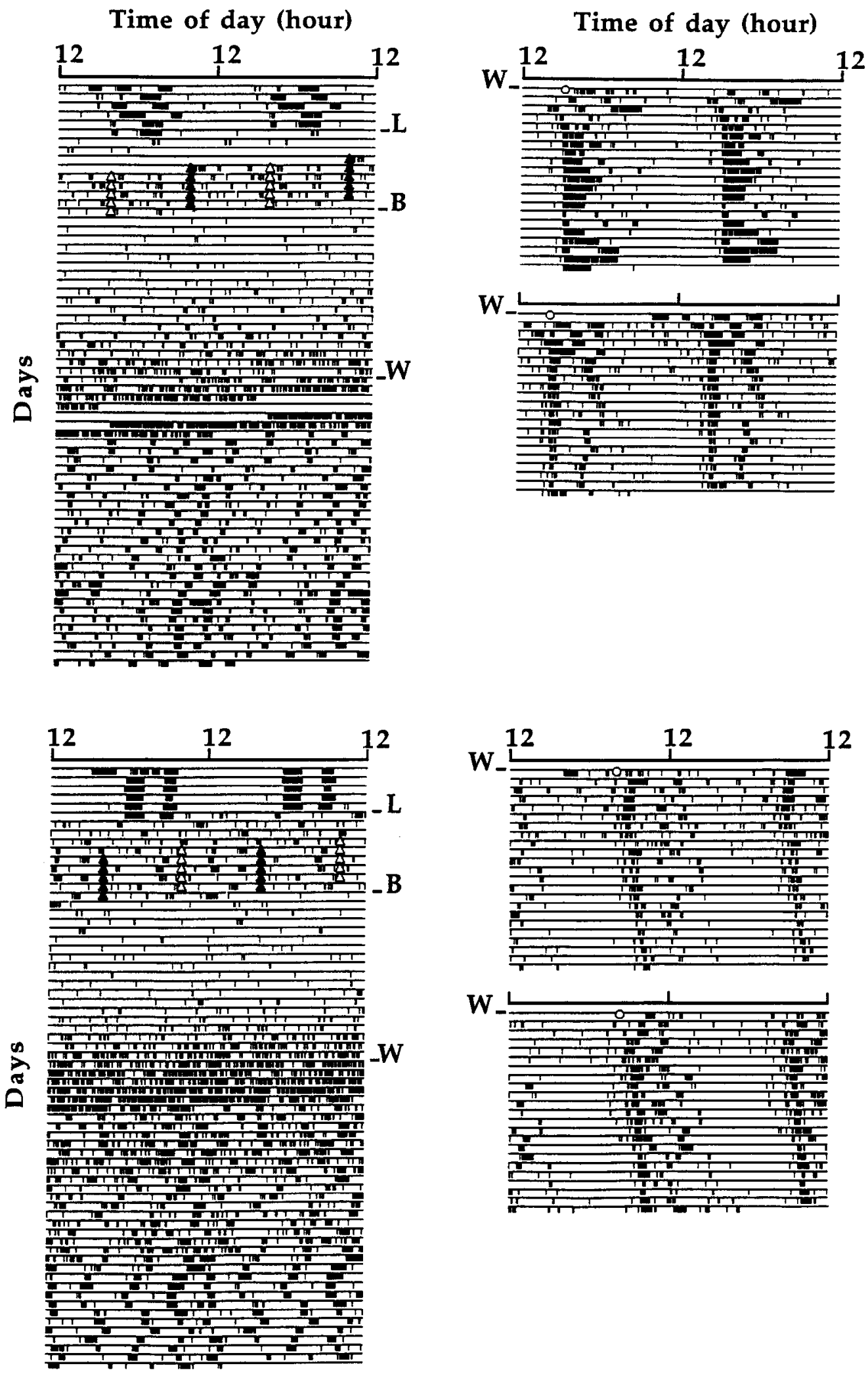

Figure 1. Double-plotted wheel-running activity records of SCN-lesioned hamsters, and the records of two pups born to each of them. The dams received SCN lesions on day 7 of gestation $(L)$ and two injections/day on days 11-15 (A, SKF 38393; $\triangle$, vehicle only; $B$, birth). The dam on the top received SKF 38393 at $0800 \mathrm{hr}$ and that on the bottom received the drug at $2000 \mathrm{hr}$. The time of activity onset for each pup on the day of weaning $(W)$ is indicated on the pup's record (O). Some pups from whom activity was not recorded remained with the dams for several days beyond the indicated day of weaning (W).

\section{Results}

Experiment 1: circadian rhythms

Figure 1 shows the wheel-running activity records of two SCNlesioned hamsters that received SKF 38393 either in the morning or in the evening, and the records of two pups born to each

of them. Although the activity records of the two SCN-lesioned dams were similar, the phases of the pups born to them were different by about $12 \mathrm{hr}$. In Figure 2, the phases of all pups in the study are plotted on a circle representing the day of weaning. The prenatal injections of SKF 38393 unambiguously set the phases of the pups' circadian rhythms. Within each of the two 

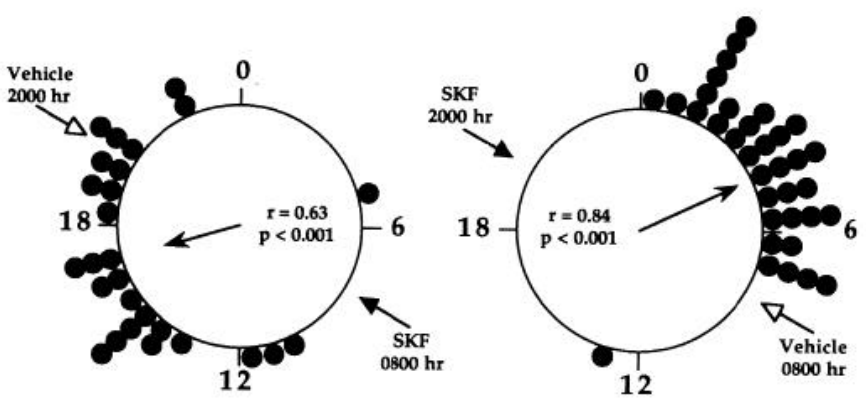

Figure 2. The phases of pups' activity rhythms on postnatal day 20 . The large circles represent $24 \mathrm{hr}$ and the small filled circles are the phases of individual pups. The distribution on the left is from pups born to dams that received SKF 38393 in the morning (0800) and vehicle alone in the evening. The distribution on the right is from pups whose dams received the drug in the evening $(2000)$ and vehicle alone in the morning. Arrows inside the circles indicate the average phases of the distributions. The synchrony within each group is indicated by the length of the arrow and the value of $r$. The two distributions were significantly different from each other.

treatment groups the distribution of phases was significantly different from uniform $(p<0.001)$. In addition, the distributions were significantly different from each other $(p<0.001)$. Prenatal administration of SKF 38393 at 0800 established an average phase in the pups of $16.87 \mathrm{hr}$ while the administration of SKF at 2000 established an average phase of $4.17 \mathrm{hr}$, a difference of $11.3 \mathrm{hr}$. This difference cannot be attributed to anything other than the $12 \mathrm{hr}$ difference in the timing of the prenatal injections of SKF 38393, since all of the pregnant hamsters were handled and received injections at both of the treatment times each day. Within each of the treatment groups, pups weaned at 0800 showed average phases similar to those of pups weaned at 2000 (drug at 0800 and weaned at 0800 , average phase $=$ $16.73 \mathrm{hr}$; drug at 0800 and weaned at 2000 , average phase $=$ $17.05 \mathrm{hr}$; drug at 2000 and weaned at 0800 , average phase $=$ $4.91 \mathrm{hr}$; drug at 2000 and weaned at 2000 , average phase $=3.34$ hr).

\section{Experiment 2: in situ hybridization}

Administration of SKF 38393 to pregnant hamsters on day 15 of gestation in either the morning or in the evening induced $c$ - fos gene expression in the fetal SCN (Figs. 3, 4). A hybridization signal was not seen following treatment with vehicle only, demonstrating a specific activation of $c$-fos expression in the fetal hamster SCN by SKF 38393. The average relative optical densities of SCN from fetuses whose dams received SKF 38393 were significantly greater than the average relative optical densities of those whose dams received vehicle only (Fig. 4). In addition, there was no overlap in individual optical density values between the drug (1.93-3.08) and vehicle-only groups (1.16-1.65). Induction of $c$-fos by SKF 38393 occurred regardless of the time of day of administration (Fig. 4). SKF 38393 induced $c$-fos expression in the fetal caudate-putamen, piriform cortex, and endopiriform nucleus (Fig. 3). The entire brain was not examined.

Film autoradiograms of sections hybridized with the $D_{1}$ receptor antisense cRNA probe revealed that $D_{1}$ receptor mRNA was expressed in the fetal SCN, caudate putamen, and cerebral cortex on day 15 of gestation in all of the experimental groups (Fig. 3). For both $c$ - fos and the $\mathrm{D}_{1}$ receptor mRNA, sense

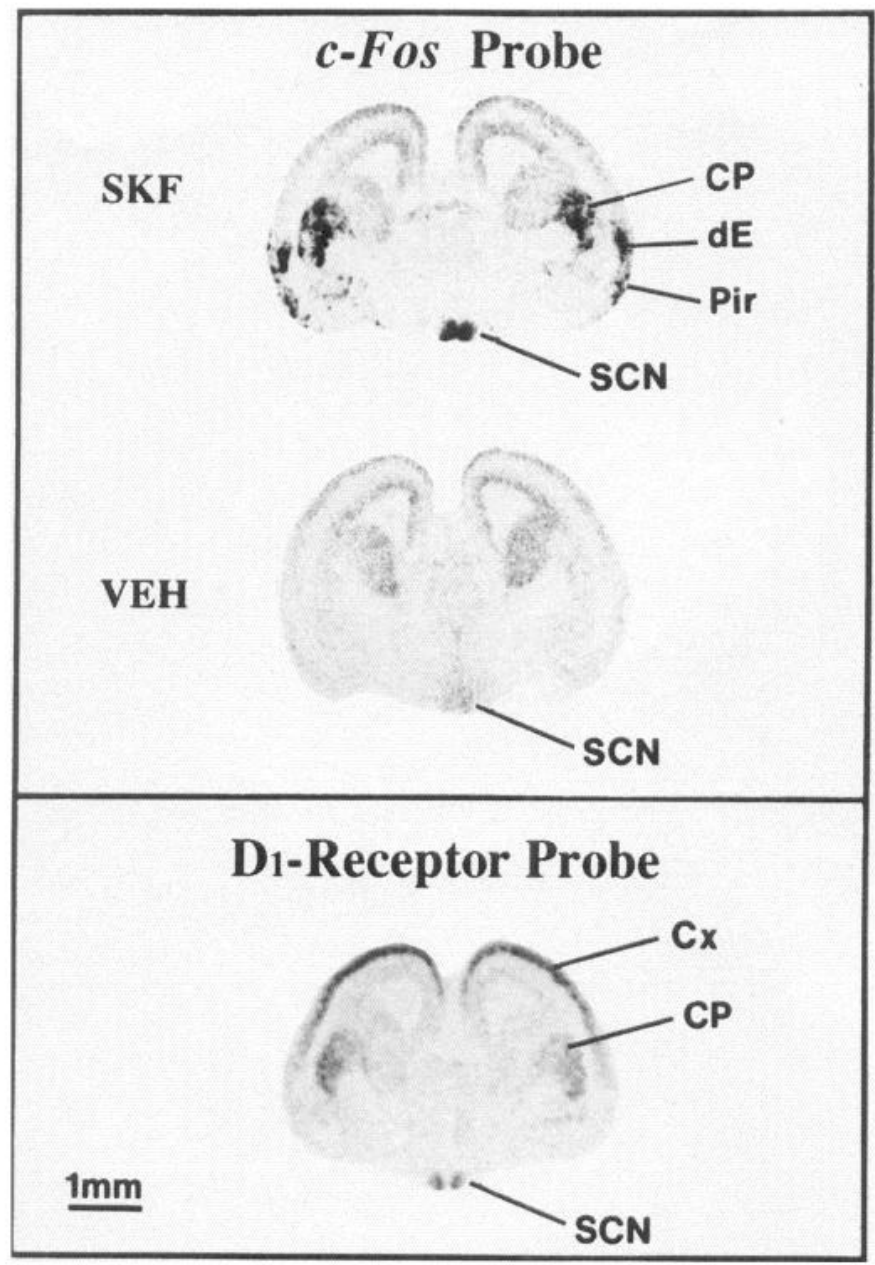

Figure 3. Representative autoradiograms of fetal (E14.75) hamster brain sections processed for in situ hybridization. Hybridization signal appears black. Top, Hybridization of the $c$-fos antisense cRNA probe to a section from a fetus whose dam received SKF $3839340 \mathrm{~min}$ before tissue collection. Middle, Hybridization of the $c$-fos antisense cRNA probe to a section from a fetus whose dam received vehicle. Fetuses from SKF 38393-injected dams showed increased $c$-fos gene expression in the SCN, caudate-putamen, piriform cortex, and dorsal endopiriform nucleus when compared to fetuses of vehicle-injected dams. Bottom, $\mathrm{D}_{1}$-dopamine receptor gene expression in the fetal hamster brain detected using an antisense cRNA probe generated from the human $D_{1}$ -

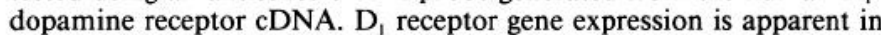
the SCN, caudate putamen, and cerebral cortex dorsal to the rhinal fissure. $S C N$, suprachiasmatic nucleus; $C P$, caudate-putamen; $C x$, cerebral cortex; $d E$, dorsal endopiriform nucleus; Pir, piriform (olfactory) cortex.

(control) probes from the same plasmids that produced the antisense probes revealed a homogeneous background of nonspecific hybridization throughout the sections of fetal brain examined.

\section{Discussion}

Prenatal administration of the $\mathrm{D}_{1}$-dopamine receptor agonist SKF 38393 to pregnant hamsters sets the phase of (entrains) the pups' activity/rest rhythms. Because pregnant hamsters received injections twice a day, the only difference between the two treatment groups was the $12 \mathrm{hr}$ difference in the timing of exposure to SKF 38393, indicating that the drug was the specific cause of the $11.3 \mathrm{hr}$ difference in average phase. It is likely that entrainment by prenatal SKF 38393 was the result of an effect 
on the fetal SCN since the circadian pacemaker, which regulates overtly expressed rhythms, such as the activity/rest rhythm, is located within the SCN. A direct effect of SKF 38393 is supported by the observed induction of $c-f o s$ mRNA and by the presence of $D_{1}$ receptor mRNA within the fetal SCN. These results are consistent with the hypothesis that maternal entrainment of the fetal circadian pacemaker is normally mediated by maternal activation of a dopaminergic system in the fetal hypothalamus (Weaver et al., 1992). Further work will be required, however, to demonstrate a specific, physiological role for dopamine receptor activation in maternal entrainment.

The induction of $c$-fos expression in the fetal hamster SCN by SKF 38393 is similar to results previously reported in rats (Weaver et al., 1992). The induction of $c$-fos and the entrainment of circadian rhythms in hamsters by SKF 38393 reported here suggest that $c$-fos induction is involved in the mechanism by which a phase-setting signal affects the pacemaker. A role for $c$ - fos induction in the regulation of circadian rhythms has been previously indicated in adult rodents (Takahashi et al., 1993). A light pulse that shifts the phase of circadian rhythms in mature animals also causes an induction of $c$-fos $\mathrm{mRNA}$ and expression of Fos-like immunoreactivity within the SCN (Rea, 1989; Aronin et al., 1990; Earnest et al., 1990; Kornhauser et al., 1990; Rusak et al., 1990).

The induction of $c$-fos by SKF 38393 in the fetal SCN may differ from $c$-fos induction by light in adults in that $c-f o s$ induction by SKF 38393 does not appear to depend on the circadian time when the drug is administered. In the case of light, $c$-fos is induced only at circadian times when light causes phasc shifts (subjective night). In the present study, SKF 38393 was administered at two times of day, $12 \mathrm{hr}$ apart, but at both of these times the drug was equally effective in both the induction of $c$-fos gene expression and causing entrainment.

Because the pregnant hamsters in these experiments received SCN lesions on day 7 of gestation, it is likely that the fetal circadian pacemakers were not entrained by the dams at the time of drug administration (E11-E15) (Davis and Gorski, 1988). Therefore, circadian oscillations generated within the fetal SCN might have had different phases in different fetuses when the drug was given. If only certain phases were sensitive to SKF 38393, then many fetuses should not have been affected, leaving significant overlap between the distributions of phases in the two treatment groups. Such overlap was not observed, indicating that all or most of the fetuses were affected by the drug. Furthermorc, there was no overlap in the optical density mcasurcments of $c$-fos induction, indicating again that all of the fetuses exposed to SKF 38393 responded.

It is possible that the generation of circadian oscillations within the fetal SCN had not yet started at the time of drug administration. If so, it is then possible that the phase-setting effect of SKF 38393 resulted from an initiation of oscillations rather than from phase shifts of ongoing oscillations. Phase setting as a result of the initiation of oscillations could account for SKF 38393 being equally effective at both of the times it was given.

It is likely that SKF 38393 acted directly on the fetus since mRNA for $D_{1}$ receptors was found in the fetal SCN. In addition, the dam is clearly not required for the induction of $c$-fos in the fetal rat SCN; direct injections of cocaine to cesarean-delivered rat fetuses caused $c$-fos induction (Weaver et al., 1992). Because few synapses are present within the SCN of rats and hamsters before birth (Lenn et al., 1977; Moore and Bernstein, 1989; Speh and Moore, 1990), it is unlikely that SKF 38393 caused

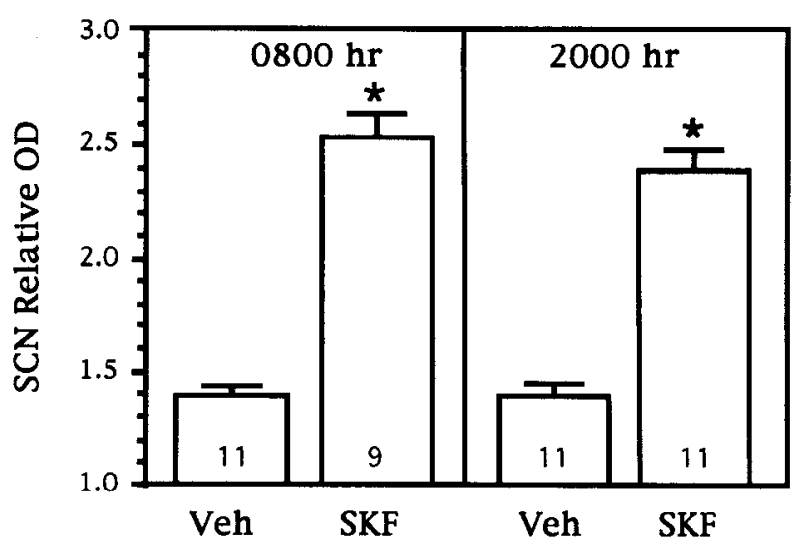

Figure 4. Relative optical densities of fetal SCN following in situ hybridization with the antisense $c$-fos $\mathrm{CRNA}$ probe. Optical densities of fetal SCN from SKF 38393-injected $(S K F)$ dams were significantly greater than those from vehicle-injected $(V e h)$ dams. A similar difference was seen whether the injections were given in the morning (0800) or in the evening (2000). Error bars indicate standard errors and the numbers of fetuses examined in each group are indicated within the columns. two-way ANOVA: treatment, $p<0.0001$; time of treatment, $p>0.3$; interaction, $p>0.3{ }^{*}$, Student's $t$ test: SKF vs Veh, $p<0.0001$.

c-fos induction within the SCN by acting on some other part of the fetal brain which then trans-synaptically affected the SCN. It is also unlikely that SKF 38393 entrained the fetal pacemaker by first entraining circadian rhythms of the dam since the dams in this study werc all SCN lesioncd. Although there was no detectable effect of SKF 38393 on the dams' wheel-running activity pattern, indirect entrainment by SKF 38393 via an effect on some other aspect of the dams' physiology cannot be excluded.

The physiological mechanism(s) by which maternal circadian rhythms normally entrain the fetal circadian pacemaker is not known. Ablation of the maternal SCN during gestation disrupts entrainment (Reppert and Schwartz, 1986a; Davis and Gorski, 1988), but other extirpations such as pinealectomy or hypophysectomy do not (Reppert and Schwartz, 1986b). Two candidate maternal signals have been identified by experimentally inducing rhythms in SCN-lesioned dams. In hamsters, prenatal injections of melatonin cause entrainment of the offspring (Davis and Mannion, 1988; Viswanathan and Davis, 1993), and in rats a restricted feeding schedule imposed on the dam causes cntrainment (Weaver and Reppert, 1989). These results suggest that there is more than one maternal rhythm that mediates entrainment of the fetal pacemaker. It is possible that the activation of a dopaminergic system within the fetal hypothalamus is a common pathway for several signals including melatonin and restricted feeding. Alternatively, melatonin and SKF 38393 may act through different mechanisms to cause entrainment since the average phase established by melatonin injections is different from that established by injections of SKF 38393. For example, melatonin administered in the morning sets phases to times in the morning (Davis and Mannion, 1988; Viswanathan and Davis, 1993), while SKF 38393 in the morning sets phases to times in the evening (Fig. 2).

In summary, entrainment by prenatal SKF 38393 and the correlated induction of $c$-fos gene expression in the fetal SCN indicate that dopamine activation affects the fetal circadian pacemaker. Further cvidence for this is the partial blockage of cocaine-induced $c$-fos expression by the $\mathrm{D}_{1}$-dopamine receptor 
antagonist SCH 23390 in fetal rat SCN (Weaver et al., 1992). The activation of a dopaminergic receptor system in the fetus may be a transient mechanism for entrainment that is lost during postnatal development; the induction of $c$ - fos by SKF 38393 does not occur in adult rat SCN at the circadian times examined (Weaver et al., 1992). Entrainment by dopaminergic activation may be part of a maternal entrainment mechanism that is no longer required after development of the retinohypothalamic tract (Stanfield and Cowan, 1976; Mason et al., 1977; Güldner, 1978; Speh and Moore, 1988, 1990) and the capacity for entrainment by light (Duncan et al., 1986), both of which occur postnatally. Although a transition in the signals that cause entrainment appears to occur during mammalian development, these signals may share common cellular mechanisms such as the induction of immediate-early genes.

\section{References}

Aronin N, Sagar SM, Sharp FR, Schwartz WJ (1990) Light regulates expression of a Fos related protein in rat suprachiasmatic nuclei. Proc Natl Acad Sci USA 87:5959-5962.

Batschelet E (1981) Circular statistics in biology. New York: Academic.

Davis FC (1989) Use of postnatal behavioral rhythms to monitor prenatal circadian function. In: Development of circadian rhythmicity and photoperiodism in mammals (Reppert SM, ed), pp 45-65. Ithaca: Perinatology Press.

Davis FC, Gorski RA (1986) Development of hamster circadian rhythms: prenatal entrainment of the pacemaker. J Biol Rhythms 1:77-89.

Davis FC, Gorski RA (1988) Development of hamster circadian rhythms: role of the maternal suprachiasmatic nucleus. J Comp Physiol [A] 162:601-610.

Davis FC, Mannion J (1988) Entrainment of hamster pup circadian rhythms by prenatal melatonin injections to the mother. Am J Physiol 255:R439-R448.

Deguchi T (1975) Ontogenesis of a biological clock for serotonin acetyl coenzyme A $N$-acetyltransferase in pineal gland of rat. Proc Natl Acad Sci USA 72:2814-2818.

Duncan MJ, Banister MJ, Reppert SM (1986) Developmental appearance of light-dark entrainment in the rat. Brain Res 369:326330.

Earnest DJ, Iadarola M, Yeh HH, Olschwka JA (1990) Photic regulation of $c$-fos expression in neural components governing the entrainment of circadian rhythms. Exp Neurol 109:353-361.

Güldner F-H (1978) Synapses of optic nerve afferents in the rat suprachiasmatic nucleus. I. Identification, qualitative description, development and distribution. Cell Tissue Res 165:509-544.

Klein DC, Moore RY, Reppert SM (1991) Suprachiasmatic nucleus: the mind's clock. New York: Oxford UP.

Kornhauser JM, Nelson DE, Mayo KE, Takahashi JS (1990) Photic and circadian regulation of $c$-fos gene expression in the hamster suprachiasmatic nucleus. Neuron 5:127-134.

Lenn NJ, Beebe B, Moore RY (1977) Postnatal development of the suprachiasmatic nucleus of the rat. Cell Tissue Res 178:463-475.

Mason CA, Sparrow N, Lincoln DW (1977) Structural features of the retinohypothalamic projection in the rat during normal development. Brain Res 132:141-148.

Moore RY (1991) Development of the suprachiasmatic nucleus. In: Suprachiasmatic nucleus: the mind's clock (Klein DC, Moore RY, Reppert SM, eds), pp 391-404. New York: Oxford UP.
Moore RY, Bernstein ME (1989) Synaptogenesis in the rat suprachiasmatic nucleus demonstrated by electron microscopy and synapsin 1 immunoreactivity. J Neurosci 9:2151-2162.

Moore RY, Card JP (1985) Visual pathways and the entrainment of circadian rhythms. Ann NY Acad Sci 453:123-133.

Moore RY, Eichler VB (1972) Loss of a circadian adrenal corticosterone rhythm following suprachiasmatic lesions in the rat. Brain Res 42:201-206.

Pittendrigh CS (1981) Circadian systems: entrainment. In: Handbook of behavioral neurobiology, $\mathrm{Vol}$ 4, Biological rhythms (Aschoff J, ed), pp 95-124. New York: Plenum.

Ralph MR, Foster RG, Davis FC, Menaker M (1990) Transplanted suprachiasmatic nucleus determines circadian period. Science 247: 975-978.

Rea MA (1989) Light increases Fos-related protein immunoreactivity in the rat suprachiasmatic nuclei. Brain Res Bull 23:577-581.

Reppert SM, Schwartz WJ (1986a) The maternal suprachiasmatic nuclei are necessary for maternal coordination of the developing circadian system. J Neurosci 6:2724-2729.

Reppert SM, Schwartz WJ (1986b) Maternal endocrine extirpations do not abolish maternal coordination of the fetal circadian clock. Endocrinology 119:1763-1767.

Reppert SM, Weaver DR (1991) A biological clock is oscillating in the fetal suprachiasmatic nuclei. In: Suprachiasmatic nucleus: the mind's clock (Klein DC, Moore RY, Reppert SM, eds), pp 405-418. New York: Oxford UP.

Reppert SM, Weaver DR, Stehle JH, Rivkees SA (1991) Molecular cloning and characterization of a rat $A_{1}$-adenosine receptor that is widcly expressed in brain and spinal cord. Mol Endocrinol 5:10371048.

Reppert SM, Coleman RJ, Heath HW, Swedlow JR (1984) Pineal $N$ acetyltransferase activity in 10-day-old rats: a paradigm for studying the developing circadian system. Endocrinology 115:918-925.

Rusak B, Robertson HA, Wisden W, Hunt S (1990) Light pulses that shift rhythms induce gene expression in the suprachiasmatic nucleus. Science 248:1237-1240.

Sokolove PG, Bushell WN (1978) The Chi Square periodogram: its utility for analysis of circadian rhythms. J Theor Biol 72:131-160.

Speh JC, Moore RY (1988) Development of the retinohypothalamic tract in the rat. Soc Ncurosci Abstr 14:50.

Speh JC, Moore RY (1990) Synaptogenesis and retinohypothalamic tract (RHT) development in the hamster suprachiasmatic nucleus (SCN). Soc Neurosci Abstr 16:602.

Stanfield B, Cowan WM (1976) Evidence for a change in the retinohypothalamic projection in the rat following early removal of one eye. Brain Res 104:129-136.

Stephan FK, Zucker I (1972) Circadian rhythms in drinking behavior and locomotor activity of rats are eliminated by hypothalamic lesions. Proc Natl Acad Sci USA 69:1583-1586.

Takahashi JS, Kornhauser JM, Koumenis C, Eskin A (1993) Molecular approaches to understanding circadian oscillations. Annu Rev Physiol 55:729-753.

Viswanathan N, Davis FC (1993) The fetal circadian pacemaker is not involved in the timing of birth in hamsters. Biol Reprod 48:530 537.

Weaver DR, Reppert SM (1989) Periodic feeding of SCN-lesioned pregnant rats entrains the fetal biological clock. Dev Brain Res 46: 291-296.

Weaver DR, Rivkees SA, Reppert SM (1992) $D_{1}$-dopamine receptors activate $c$-fos expression in the fetal suprachiasmatic nuclei. Proc Natl Acad Sci USA 89:9201-9204. 